\title{
"KT" CarePacks - Collaboration Patterns for Knowledge Transfer: Learning from IS/IT- Outsourcing Case at a Swiss Financial Institution
}

\author{
Malgorzata Bugajska \\ Department of Informatics, University of Zurich, Switzerland, bugajska@gmail.com
}

\begin{abstract}
Organizations now more than ever focus on fostering team work in their daily activities to secure better results for their stakeholders. Team work and collaboration are especially important for inter-organizational outsourcing relationships where these qualities are crucial for the successful knowledge transfer conducted throughout all phases of outsourcing relationship. Knowledge workers involved in such complex, inter-organizational collaboration processes require support to secure structured and well managed collaboration. Consequently, there is a strong need of service receiver organizations to use sustainable approaches for the knowledge transfer to satisfy recurring transfer processes in forthcoming sourcing activities. Idea of "pattern" offers encapsulated approach for describing solutions for recurring problems and is already successfully used within the IT domain. In this paper we present the concept of patterns for the sustainable knowledge transfer for outsourcing relationships. We introduce CarePacks - reusable patterns for supporting act of the collaborative knowledge transfer and present lessons learned from introducing them at a Swiss financial institution while conducting six knowledge transfer pilots in three consecutive trials.
\end{abstract}

Keywords: Knowledge transfer, IS/IT outsourcing, Patterns

\section{Introduction}

Knowledge transfer is one of pthe critical factors that increases risk throughout all IS/IT sourcing relationship phases (Bloch, 2005; Carmel, 2005). It is therefore imperative to work towards achieving desired knowledge balance before deciding on a renewal, redefinition or termination of an outsourcing relationship and insource IS/IT services back home. To achieve the successful knowledge transfer between organization outsourcing IS/IT activities (the client) and the supplier of 
IS/IT services, both parties need not only a measurable and result oriented transfer process, but also ability to support their transfer teams with tools (analog and electronic alike) and processes that help to achieve anticipated transfer results.

The knowledge transfer process within an organization has been researched extensively (Davenport, 2000; Davenport, 2005; English, 2006; Nonaka, 1995; Szulanski, 1999; Von Krogh, 1998). However, there still appears to be limited research on inter-organizational knowledge transfer, although researchers do point out that it is most likely a more difficult and complex task (Darr, 2000; Kim, 2000).

To understand the inter-organizational complexity of the knowledge transfer, it is important to analyze the factors influencing the transfer of knowledge. Based on researching approximately 100 pairs of client and service providers (consulting services), researchers $(\mathrm{Ko}, 2005)$ have modeled factors influencing the knowledge transfer between the clients and consultants. They list: communication (encoding and decoding content as well as source credibility), knowledge (absorptive capacity, shared understanding or arduous relationship) and motivational (intrinsic, extrinsic motivation) factors which influence the knowledge transfer. On a more general basis, researchers conclude that frequency and depth of personto-person contact (Rulke, 2000), as well as congruency of organizational and individual goals (Jensen, 1976), play a role in defining quality of transfer within a company. Additionally, researchers describe the limitations in terms of transfer of expertise (expert to novice) which has been investigated at cognitive (availability bias, course of knowledge or "not invented here" syndrome) and motivational/intentional levels (reward systems, culture of trust) (Hinds, 2003). Consequently, understanding of organizational culture of the client and outsourcer is named as one of the most important factors of managing and deriving value from offshoring businesses (Carmel, 2005). Consequently, being aware of factors influencing the knowledge transfer it is important to use right transfer methods and tools for particular transfer situations (Davenport, 2000; Dixon, 2000). Appropriate transfer tools or instruments need to support the team of client and supplier in their transfer activities, since failing proves too expensive and risky for the client organization or significantly impedes attracting new clients in case of service providers (Carmel, 2005; Cohen, 2006).

This paper is organized as follows: in the next chapter we present the research question which we explored in the field project. Further, we describe the design methodology and data collection for our explorations. Consequently, we present the pattern approach for the knowledge transfer in the IS/IT outsourcing domain. From there we present an example of a CarePack followed by lessons learned acquired during our three implementation trials in the field (carried out by us at a Swiss financial institution, the "Institution"). We conclude by presenting practical implication for the use of pattern based CarePacks approach for the knowledge transfer. 


\section{Research question}

Contract bound knowledge transfer in IS/IT outsourcing is executed jointly and collaboratively by two organizations during all outsourcing phases. Therefore, unsurprisingly there are enormous benefits (monetary, time based as well as organizational) to be gained from facilitating repeatable transfer approaches. Such approaches are already part of the value proposition of the IS/IT service provider champions (compare IBM or XANSA for Business Process Outsourcing). These organizations are committed to understanding the processes at client organizations as quickly and accurately as possible. The service provider teams are trained for achieving maximum progress in transferring knowledge (both explicit and implicit) from the client company to their own. Furthermore, well designed and sustainable knowledge transfer during the whole outsourcing cycle, as well as mutual systems for managing knowledge, contribute significantly to the quality of the relationship, increasing the likelihood of a prosperous relationship and consequently engaging new clients for the business (Gottschalk, 2007).

However, transferring knowledge proves difficult for majority of companies outsourcing their IS/IT activities (Carmel, 2005). Often an outsourcing service recipient lacks an appropriate set of instruments and methods to make the collaborative knowledge transfer sustainable. Furthermore, their skills for sharing knowledge between their own teams and teams of the service provider are often not mature enough. Consequently, the client as service recipient often follows set of unstructured activities, which are parts of processes designed by service providers. In many cases these are the only available transfer approaches at hand so the client will likely use them. Obviously, following parts of "foreign" processes which are not designed to secure strategic goals of the client does not necessarily contribute to the successful knowledge transfer for the client's organization. Therefore, redesigning or terminating the outsourcing relationship by the client is often described as difficult (and expensive) experience for the entire organization.

Sustainable knowledge transfer is a critical success factor for building competitive advantage for organizations wanting to profit from sourcing projects and not making it a costly mistake. Successful transfer needs to be based on the inter-organizational collaboration to meet the transfer goals agreed upon in the IT/IS outsourcing contract. Therefore, there is a need for well designed collaboration processes for knowledge workers involved in the transfer process. The value of collaboration for accomplishing organizational tasks is widely known and admitted (Briggs, 2003). In inter-organizational collaboration, the complexity is significant since the goals are to be accomplished by a team with whom the members most likely do not share the culture, communication and coordination processes. The importance of teams for accomplishing sourcing projects is confirmed by research (Carmel, 2005); therefore, any support given to the teams which could improve their performance is of value to organizations. The support for the collaborating team is often provided by collaboration facilitators. Although 
this is a very good solution, it is costly and does not guarantee sustainability of the solution if the facilitator is not available. As such the challenge for the organizations is to reduce the involvement of collaboration facilitators without endangering the drop in quality of the support for the teams and securing the sustainability of the collaboration support processes. The question thus arises how to design and implement the collaborative knowledge transfer to achieve a more sustainable approach within the inter-organizational set-up. Based on the methodology of work sciences, we designed our research as explained in the following chapter.

\section{Research design and data collection}

Together with the Institution, our research partner in Switzerland, which was in the process of remodeling relationships with their long term outsourcing partner, we designed the three year research project (with a total effort of about 10 person years) called Knowledge Transfer in Outsourcing Relationship. We used this relationship as a base example for researching the knowledge transfer in IS/IT outsourcing to design the sustainable knowledge transfer. During the outsourcing relationship, the Institution and its IS/IT service provider transferred the knowledge on a "need-based" basis. Whenever there was an operative need for the transfer, the transfer was performed. Since the Institution wanted to redefine its outsourcing relationship, it also started to rethink processes concerning the knowledge transfer. The Institution committed itself towards a more sustainable and measurable process for the knowledge transfer. Currently, its outsourcing partner develops and retains maintenance of a strategically important transaction processing software bundle. This software bundle is not only used by the Institution itself, but it is also used to provide a great number of services to other banks. Therefore, our objective in this project was to design a method to moderate the sustainable knowledge transfer process and to provide tools which can be reused for dealing with recurring problems in transferring knowledge between organizations. Results reported in this paper were achieved while focusing on insourcing the knowledge from the service provider back to the client in the last phases of their outsourcing relationship. The use of the method for earlier phases had already been tested with positive results.

Our research is design oriented (Heinzl, 2001; WKWI, 1994). Its objective is to develop solutions to generic types of problems on a medium level of abstraction, e.g. in the form of an architecture (in the tradition of computer science research), a reference model (Becker, 2004), or a methodology (Braun, 2004; Heym, 1993). Therefore, we use design methodology referred to as a "theory for design and action" (Gregor, 2006), which explains "how to do" something through defining principles of form and function and methods. Contribution of such design theory is seen as "utility to a community of users, novelty of artifact, and the 
persuasiveness of claims that is effective "(Gregor, 2006). In the context of our investigation:

1) we provide the users with the utility by supplying the practitioners with the artifacts - the CarePacks to provide or improve sustainability to the knowledge transfer processes; since a thorough literature review (Bugajska, 2006; Voigt, 2007) shows that no comparable methodology has yet been developed, particularly none that relies on replicable patterns for action);

2) Our approach is new, drawing creatively from interdisciplinary research (using available frameworks (e.g.(Hutzschenreuter, 2004; Thatchenkery, 2005), methodologies (Mulder, 2007) and techniques (e.g., after action reviews, 5-why etc. see (Dixon, 2000; English, 2006)) for KT and to draw conclusions for methodology development (see also (Bugajska, 2007); The literature review uncovered only methods either for the knowledge transfer (e.g. (Davenport, 2005; Dixon, 2000; English, 2006; Nonaka, 1995)), for managing IT/IS-Outsourcing relationships (most importantly: (Carmel, 2005; Cohen, 2006; Willcocks, 2006)) or for repeatable patterns of collaboration (Briggs, 2003; de Vreede, 2006), but none in the intersection of the three;

3) Through the multiple trials/pilots and cyclical usage of the CarePacs, we demonstrate the persuasiveness of our design. In such cyclical process the "knowledge is used to create works, and works are evaluated to build knowledge" (Owen, 1997).

\subsection{Data collection}

An important starting point was to understand the demand for the transfer to uncover which knowledge (packaged as "knowledge items") needed to be transferred, what were its distinctive characteristics and understand underlying causes for existing imbalance of knowledge. Additionally, to get more insight into cultural and communication approaches taken by different teams as well as to better understand the domains of the knowledge workers, we conducted jobshadowing and visited informal meetings of communities of practice. Furthermore, evaluation of the pilots contributed to the design of the next round of pilots. Altogether there were three rounds of trials and the fourth round is currently being prepared.

Ability to define and describe knowledge considered for the transfer and define the most successful way to implement it as well as secure its strategic fit requires full attention of the outsourcing client. Therefore, the important part of the knowledge transfer processes is to analyze the demand for the knowledge transfer in the first place.

The DEAN (The DEmand ANalysis for Knowledge Transfer) methodology allows for mapping the demand for the knowledge transfer in outsourcing relationships. We used this method to define which knowledge needs to be transferred from the outsourcer back to the client organization. The DEAN 
methodology (with its five phases) is described in detail in (Bugajska, 2006); it uses "knowledge item" as the smallest package of knowledge to be transferred between organizations. Since knowledge is by definition networked, there are no natural knowledge packages and therefore the packages need to be constructed with help of definitions describing organizational tasks. The suitable granularity is usually found in job family descriptions (e.g. job family - software architect) used within the organization.

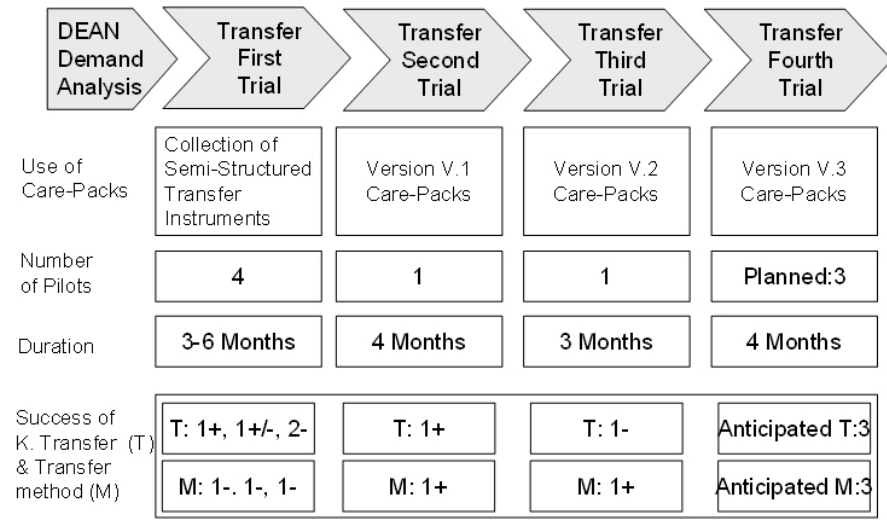

Figure 1: Summarizes the evolution of the method based approach, number of pilots, the duration of each trial as well as the success (marked with "+") of the knowledge transfer and/or the transfer method used. The reasons behind the success and failure of the approaches are described in text.

During these trials we observed how the transfer instruments, which later evolved into CarePacks, were improved and what was achieved in terms of transfer of knowledge within teams. During and after each trial we performed interviews and workshops with the main actors from both the management and user levels of the two organizations. During the 18 months of the project, the project members conducted a total of 38 (structured) interviews, 22 workshops (including 4 large group workshops using GroupSystems technology) and eight feedback sessions with 29 distinct actors including the CIO of the client and the CEO of the service provider organization. Notes were taken during all data gathering, but only some of the interviews were transcribed. In order to verify the data and to ensure objectivity to the largest extent possible, the author engaged in an additional round of six interviews immediately prior to the publication of this paper. Additionally, the author again reviewed documents form all meetings (pilot and expert meetings as well as project steering committee meetings) and corresponding meeting notes. Furthermore, the interviews conducted by a project member working on all trials have been supported with analysis of data available in (not restricted and project related) email communication streams exchanged with the Institution and its service provider. 


\section{4. "CarePacks" patterns for sustainable knowledge transfer}

To explore our research question and to address the needs of our business research partners we turned towards the growing domain of collaboration engineering. Collaboration engineering researchers stress that there is a need to structure and manage collaboration processes to make the involved individuals focus more on achieving joint goals (de Vreede, 2005). This builds on the idea of pattern language (Alexander, 1977; Alexander, 1979) which was proposed to allow for anticipating predictability of particular architectural design activity by creating patterns incorporating a description of context, problems and solutions. Building on that pattern language created for the building environment, the software engineering patterns emerged (Gamma, 1995; Lukosch, 2006) offering reusable blocs for approaching recurring problems in software creation domains. Further, this methods and approaches are used for exploring collaboration processes which are recurring in nature and proposing approach for "packaging" the experience of collaboration facilitators ("ThinkLets - "reusable, predictable and transferable facilitation techniques" (de Vreede, 2006)). Commitment towards designing routine collaboration procedures significantly helps in securing achievement of the knowledge transfer goals set by the recipient of services. Important for introducing and supporting routine behavior in the organization are collaboration instruments which can be used by employees to conduct the transfer of knowledge with reduced presence of the facilitators.

Therefore, when the management of the Institution (which was in the process of in-sourcing the knowledge back to the organization) requested a more formalized approach for preparation and execution of the various pilots currently executed in the organization the opportunity for designing a more sustainable approach for this and future transfers emerged. Our answer was to design a set of CarePacks-based patterns for preparation as well as execution of the knowledge transfer initiatives within an outsourcing relationship. The facilitator and the team leaders involved in the transfer used CarePacks for preparation and execution of various transfer pilots. The knowledge keeper (an expert in the team of service provider) and knowledge receiver (the client's team member - a novice) followed the method as described in CarePacks to deploy the pilot and effectively conduct the transfer of knowledge without the support of external facilitator. 


\section{Structure of the CarePack}

The knowledge transfer CarePack is a document which describes a method for transferring knowledge between individuals or groups within a specific transfer context (e.g. inter-organizational transfer) with particular transfer purpose or goal. The name "CarePack" was coined by the knowledge transfer project leader from the Institution and was quickly accepted by the organization and our research department. The CarePack is characterized through its structure including exemplary usage and description of resulting organizational context emerging after deployment of the CarePack. The CarePack reflects well the idea of engineered value of documented procedures created for sustainable care, maintenance and support for the organizational wide knowledge transfer initiatives. The CarePack is structured as follows:

Context Context of the CarePack usage - a description of Real-World Scenario (e.g. preparation and Name for the transfer or transfer implementation).

Goal of the CarePack (what will be achieved) and its addressees (who may use it)

Forces What event triggered the usage of this CarePack? (e.g. change of service's supplier)

What are the collaboration principles of the CarePack? (e.g. on-site but asynchron online meetings)

How use of the CarePack supports confidence and trust in transfer team?

What factors support collaboration processes in transfer teams?

How is the collaborative goal achievement controlled?

What is the contribution to the sustainability of knowledge transfer process?

Problem Transfer problems and issues (e.g. transfer of knowledge from individual "memories" to the group "memory")

Purpose and transfer goal of this CarePack

Solution Core Idea:

Factors dominating the use of CarePack

Applicability - When to use this CarePack?

Non-applicability of the CarePack .

Procedural description of the CarePack (step-by-step)

Link to templates and guidelines supporting the execution of this CarePack (and which has already been used within the organization)

Involved actors: e.g.: knowledge keeper, knowledge receiver, transfer team leader.

Use of artifacts/tools: e.g. Blog, Competency Development tools.

Cooperation and Collaboration processes for transfer team members

Tips: (Solution applied)

Here the tips for introducing this CarePack within the organization are presented. E.g.: how to deal with possible lack of managerial engagement or what can be used to help coworkers to deal with uncertainness of discussing taboo issues.

E - tools E-Tools supporting CarePack usage (e.g. Learning Diary-Blog, WIKIs, SharePoint space) 


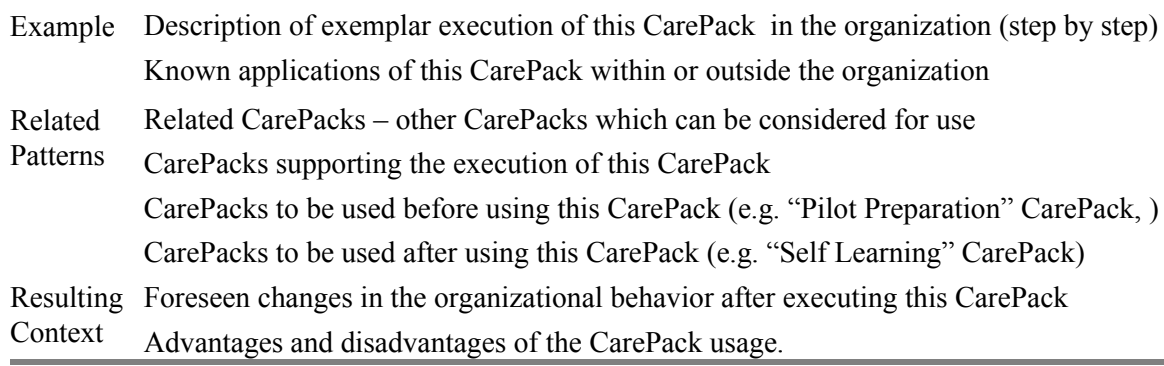

Table 1. Structure of the CarePacks for the knowledge transfer process in outsourcing relationships.

As presented in Table 1, the CarePack structure carries the elements of the pattern already proposed by Alexander (Alexander, 1979) and used by Gamma (Gamma, 1995). A CarePack is identified by its name and contains a brief context of its usage, a description of the recurring problem that it can be applied to, a proposed solution and a resulting context. Additionally, we list the forces shaping the transfer situation for which we suggest to use a particular CarePack. A CarePack document also includes other elements which are the standard components of internal documents within many organizations, such as an explanation of used terms and abbreviations or other referred documents (e.g., links to organizational library of processes). It is important to mention that CarePacks always offer "Tips" (particularly important in the solution section) along the document which provide a practical link between an abstract content of the document and real-world situations of the outsourcing-based knowledge transfer. "Tips" are often derived from organizational "lessons learned" or "best practices" created or collected while using CarePacks.

The structure of the CarePack evolved during the trials performed at the Institution. We enhanced it significantly after the third trial adding: "Collaboration Principles of the CarePack", "Electronic Tools" and collection of known "Triggering events" for deploying the CarePack (forces of the CarePack). Collaboration Principles are introduced to support the collaborative character of the knowledge transfer. This should help not only to better understand the effect of collaborative work in achieving the transfer goals, but also determine how such collaboration can be better planned or deployed in the future.

The concept of "Resulting Context" which is often used in a pattern structure is an important element for understanding what can be anticipated after certain CarePacks are used for the transfer. Usage of certain CarePacks can lead to the improvement of specific skills in members of the team and thus impact the triggering of the organizational change process which needs to be addressed.

Further, we plan to enrich the CarePack documentation with links to other teams within the Institution which have already successfully used a CarePack or collected best practices of using particular CarePack for a particular knowledge transfer. This may improve the matching of the CarePack to a particular transfer 
situation and enrich the list of ill-suited usage of a particular pattern for the industry.

\section{Example: "Project Reflection" CarePack}

CarePacks are particularly effective for teams in outsourcing relationships since they offer a structured and detailed approach for the transfer team (or teams). yet they are still flexible enough to be re-modeled and improved to suit a particular context or situation. Consequently, as a consecutive user of a certain CarePack the transfer team profits from the information about the previous usage of the CarePack and its possible modifications. Here we explain how the use of CarePack is incorporated into the transfer process and further present the "Project Reflection" CarePack in more detail. This is one of twelve CarePacks we designed and used in the field (some of the others include Buddy Support, Self-Study, Tandem or Management Coaching CarePack).

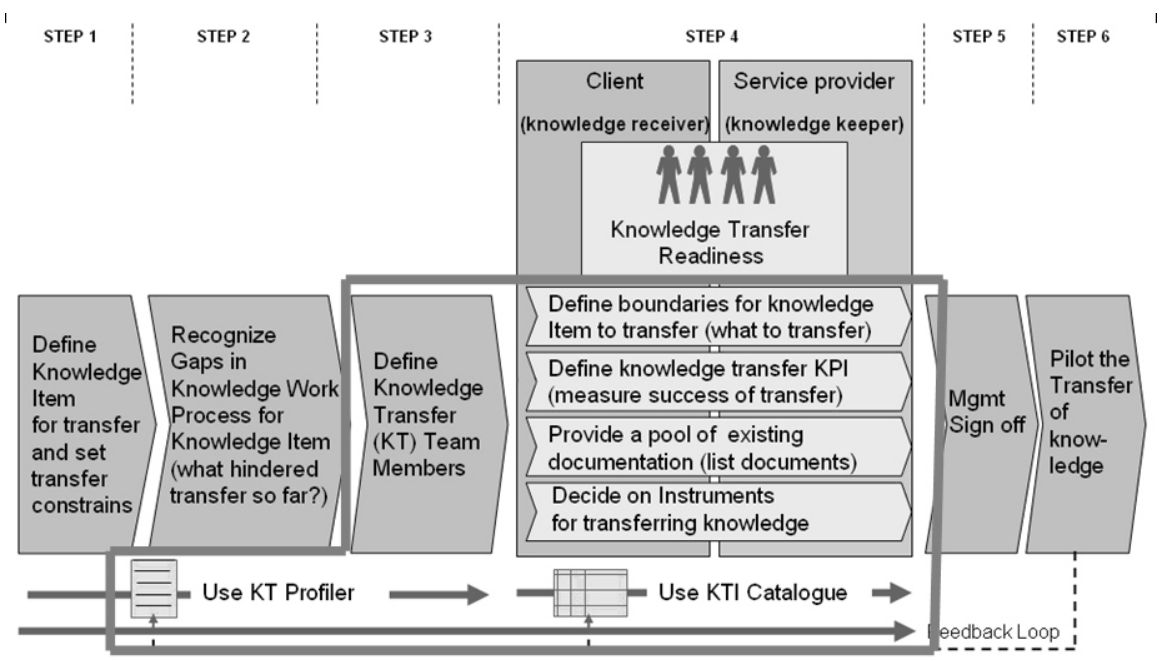

Figure 2: Process of designing pilots for the knowledge transfer in IS/IT outsourcing relationship as proposed by (Bugajska, 2007). The procedures of steps 3 and 4 as well as tools (KT Profiler and KT Instrument Catalogue) can be defined in form of a CarePack for reusable and therefore more sustainable knowledge transfer procedures within organization.

Consider following scenario: A LocalBank sets up an outsourcing project and invites a SoftwareHouse firm to support their own IT department to develop crucial software for bank operations. After some years of partnership a service provider has an immense knowledge about the design of this software. However, the bank IT team was never really involved in the design part of the software and 
their knowledge about the further development of this software does not allow them to continue this project without external support. Furthermore, the skills needed to perform more sophisticated software coding are not any more available in the bank team and have to be regained or bought. As the bank needs to regain the knowledge and starts to in-source back the knowledge from their service provider, the bank team - now in the role of "a novice" - needs to gather more expertise in activities so far performed by the service provider. Apart from documentation (its transfer or creation) - a transfer of tacit knowledge (experience) is essential. The service provider has been leading projects and took responsibility for the timely delivery of the service (e.g. software updates, design of new software modules) as well as appropriate distribution of tasks during the projects (including tasks for the bank team). Both teams need to participate in the knowledge transfer projects with well designed goals of completing a transfer (e.g. design of sub-module of software). The transfer process needs to be constantly present and should not be reinvented every time there is a need for transfer. It means that the group of professionals has to now incorporate another set of activities to their daily schedule of the software production - activities which from now on are part of the organizational routine for both organizations. The organization uses CarePacks to support their transfers and support the transfer routine.

\section{Example: "Project Reflection" CarePack}

Context and name: Project Reflection CarePack is to be used shortly after a jointly (client and service provider) executed project to reflect on the transfer of knowledge between client and service provider which has been performed in such a project. This pattern supports structured exchange of insights among members of the project. This CarePack is based on the transfer instrument called After Action Review which was developed by and for the US Army (Dixon, 2000) to help the individuals involved in a group activity to share knowledge among project members and contribute to the organizational memory of the project team after "the action" (often a military based field intervention) took place.

Forces: Use of this CarePack is triggered by the need of sharing views about the knowledge transfer in a project in the open forum consisting of all involved project members. The collaboration principles include: openness toward views of others, contribution towards a much richer "group view" and also understanding value of the team based improvement. Confidence and trust in the transfer team is strengthened through the project members' joint creation of the view on the project without imposing any hierarchical and organizational factors on the members expressing their point of view. Collaborative goal achievement is controlled through the level of active participation during Project Reflection meeting. This CarePack contributes to sustainability of the knowledge transfer through imposing a behavior of sharing during specially designed meeting where 
the meeting results are available immediately and the team (consisting of the client and service provider members) is actively involved in the process.

Problem: To understand if the knowledge transfer project (or project module) accomplished what was planned and if not, to understand what accounted for a change. This is a collectively built understanding where members of both the client and its service provider project members (two different organizations) need to contribute jointly.

\section{Solution:}

The knowledge transfer leader or a project leader prepares a collaborative meeting which leads towards building an understanding of transfer activity just performed. Such meeting takes place in a critical time/space of the collaborative work - which is right after completion of a particular jointly executed project. This is often a moment preceded by a considerable stress with great temptation for not to prolong the project through additional activities regardless of the achieved results. On the other hand, this is also a great moment for a group based reflection. The pursued project activities are still fresh and involved actors are still available for discussion and opinion. Therefore, the "Project Reflection" meeting is an essential part of wrapping up the knowledge transfer activities. It needs to be carefully prepared and communicated. Such meeting requires a presence of all the actors involved in this multi-organizational project. The procedural description of the CarePack describe step-by-step how such meeting need to be organized what artifacts are needed for the meeting, agenda, activities and the tips for the moderator. It is important to make sure that all the involved actors are invited (using communication guidelines of respective organizations), that they understand the goal of the meeting, are aware of the meeting agenda (3-Point agenda), understand and follow the rules of the meeting (explained below), and can actively participate in the meeting. The goal of the meeting is to build a shared understanding about the project completed by a team consisting of a service provider and client organization. Therefore, it is crucial that all the members of the project can describe transfer activities (and issues) as they have experienced them without the need to consider company (or particular group's) politics, fear of losing a face in the company or losing a job. This kind of a meeting does not require "meeting minutes", however a personal note taking is allowed. Through sharing different views on particular issue or activity the whole group can better understand the complexity or origins of problems which can be avoided or redesigned in the future. Furthermore, particularly successful decisions can be validated and supplied as a guideline for further use in the organization(s). The project leader may point out a person which helps the meeting members to stick to the rules set up for this meeting.

This CarePack can not be used to collect information which is to be used against the subordinates as a proof of their wrong-doing or possible mistakes of a particular individual during the project.

E-tools: This CarePack procedures are to be used during face-to-face, synchronous discussions. However, if face-to-face meetings can not be conducted 
the transfer team may decide to use electronic communication tools. It is important to remember that use of electronic tools often allows for a very detailed way of tracing the comments to their authors and for this reason the comfort of open information sharing in this context needs to be provided.

\section{Example and Related Patterns:}

Example: The teams of both outsourcer and service provider are involved in a project which provides a company with a semi-annual update (release) of a crucial software (adding functionality and improving software performance). Part of this project is to transfer knowledge about the particular software module. After the new software release is implemented the team meets to discuss following aspects:

- what was planed to be transferred during this project (knowledge about the software module which allows the novice to create new libraries)

- what was actually achieved (new libraries are created but there is a loose of all old ones - a costly mistake). The team discusses what accounted for a change: expert believes the novice made a fatal mistake and was aware of the risk; the management of both teams think that there is a need for a better "safety net" for a novice working on a software module; the knowledge receiver is convinced that a novice took the risk but he was not aware of the consequences.

The team improves the transfer procedures and makes sure that both the novice and the expert are still able to perform joint activities.

Related CarePacks are listed; e.g. "Transfer Preparation" CarePack with information and procedures for successful start of the transfer pilot projects which, among others includes communication guidelines for organizations involved in outsourcing project. Use of "Transfer Preparation" CarePack results in the choice of appropriate group of knowledge keepers and receivers, identifies responsible management, identifies knowledge items for the transfer, defines time and methods for such transfer and provides a document which includes this information and is signed by the management of both organizations.

Resulting Context section lists advantages and disadvantages of this CarePack. A clear advantage of this CarePack is its structured approach used for enriching a group memory through group based reflection conducted after completing the project involving the knowledge transfer. A disadvantage for this CarePack is that if the project members are not able to follow rules of the meeting as described in the CarePack (e.g. forget about the organizational hierarchy while discussing the outcomes of the project) there is a possibility the team may face more challenges than before the meeting.

This CarePack facilitates the structured approach for: understanding what was the knowledge transfer supposed to achieve, assessing if it was achieved and identifying what accounted for a change during a jointly executed project. It supports the project leaders in designing a meeting with a " 3 - Points" meeting agenda and offering a set of rules for participants to support an open exchange of insights and views. Furthermore, the tips collected by previous "users" and added to the CarePack help to deal with e.g. particular "organizational culture" related issues experienced by the participants in previous meetings. Furthermore, it 
supports the enhancement of organizational memory of the teams helping them performing better in the future.

\section{Introducing CarePacks at a Swiss financial institution}

We had an opportunity to conduct three trials and we worked on improving the design methodology for the sustainable knowledge transfer at a Swiss financial institution. Here we report our observations and first lessons learned from designing and implementing CarePacks-patterns for the knowledge transfer in outsourcing relationships:

\section{First trial:}

Introduced artifacts to support the transfer: In our first trial we used "Knowledge transfer instrument catalogue". Based on the extensive literature research we created a catalogue of "methods" explaining how the knowledge (tacit, implicit or explicit) can be transferred between groups or individuals and also what qualities of involved individuals are needed for successful transfer (Bugajska, 2007). Transfer instruments can be accessed at: www.swissprimary.ch. Furthermore, we offered first electronic support for the transfer teams e.g.: "Online learning diary" (in form of a Blog) to describe the transfer process. Additionally, "wiki" space for the transfer team was created to foster sharing of information about knowledge items which are to be transferred together with contacts and repository for transfer related e-documents.

What we have learned: Catalogue of methods for the knowledge transfer is a great tool for team leaders to help propose appropriate instrument to their transfer teams. However, a relatively short description of the instrument did not suffice to convince or engage the team to implement it for their field activities. It offered too much "open space" without precise directions. Furthermore, while working on several pilots simultaneously, we (the transfer facilitators) realized that there is a strong need to define routine approaches for the same activities (e.g. transfer preparations, procedures for choosing a transfer method, complementing with communication processes of both organizations). Consequently, proposed e-tools were not used effectively. One e-tool called "Online learning diary" (offered as an online Blog) was not used willingly due to the lack of openness of one's activity toward the group. Furthermore, "wiki pages" introduced as a collaboration platform to describe knowledge items for transfer (to be used by both organizations) were still not user friendly enough. Overall, the introduced activities were perceived as considerably slowing down operational activities. Additionally, interview conducted with one knowledge receiver revealed the existence of transfer instruments created by the knowledge receiver himself which (unfortunately) ended up hidden from the rest of the team (and not shared). This clearly shows that the transfer between organizations needs to be connected to the 
internal knowledge management processes and needs to inform internal management of the improvements realized as part of the process.

What we addressed: We proposed to introduce a more formalized approach for the knowledge transfer. We proposed a contract like agreement which included transfer goals, results and measurements (KPIs) for each knowledge transfer item and the involved transfer team. Furthermore, we offered an approach for internal knowledge build-up as an alternative for transferring the knowledge (e.g. internal trainings or "buying" the knowledge keeper). Moreover, we concentrated on designing a more sustainable approach for conducting the knowledge transfer without the constant presence of a transfer facilitator during the transfer meetings. Additionally, we built an organizational support for the idea of a more collaborative (client and service provider) knowledge transfer. This includes offering more visibility for the transfer team through the appropriate design of the transfer pilot. The transfer team needs to be recognized as a contributor to the overall group of the whole software development unit and, as such, receive adequate support for contributing to the group innovation and improvement of group performance.

Second Trial:

Introduced artifacts to support the transfer: We used set of CarePacks for preparation and execution of the knowledge transfer. We created more user friendly "wiki" pages and included an example for future users. We introduced a process for automatic upload of a "Learning Diary" which the user completes on his computer and share with group when he finishes it.

What we have learned: The collaboration requires a structured approach to support the transfer involving people representing different organizations and also find ways to support the client organization in distributing the knowledge across the team members. Moreover, successful transfer of knowledge requires collaboration between the knowledge keeper and knowledge receiver and it is important they take active part in designing the transfer. For example, one of the knowledge keepers reported that our self study template needed to be remodeled. He proposed his own template (for learning the software) which soon was accepted by the knowledge receiver. Such "filled" template is now used as a software-developer handbook by the team. From the perspective of use of patterns, we saw it as an interesting approach for using the pattern as a creative redesign and re-definition of routine processes to address the needs of the organization.

We also learned that the roles of motivator and transfer sponsor taken by the team leader were essential for the success of the knowledge transfer initiatives. Important element of the transfer was the choice of the CarePack to support the transfer team according to the personal characteristics of the knowledge keeper and receiver. Furthermore, linking the knowledge transfer initiatives to internal knowledge management processes requires that middle management understands well their challenges in the process of transferring knowledge within organization ("Middle-up-Down Mgmt Style”, (Nonaka, 1995)) 
What we addressed: We offered design approach for improving the transfer processes by introducing the CarePacks patterns of which structure accepts and invites their re-design. For the CarePacks, we decided to introduce a grid of clearly defined milestones allowing the team leader to check if the knowledge receiver is still on track. Often, if the transfer is successfully accomplished, the knowledge receiver (novice) becomes an expert of some sort. Therefore, it is important to reflect that in the talent development process within the organization. Furthermore, successful transfer reflects the ability of the receiver to absorb the knowledge under given circumstances.

\section{Third Trial}

Within this trial we introduced "train the trainer" initiatives for the Knowledge Transfer Coach as well as Coaching for middle-managers to act on their "Middleup-Down Mgmt Style" for more successful knowledge transfers.

What we have learned:

Only some portion of the process becomes "the regular and predictable behavioral patterns $[\ldots]$ that are coping with a world of complexity and continuous change" (Pavitt, 2002). Therefore, the role of KT Method Coach is to recognize them and to deal with the rest of the situations which shape the uniqueness of every knowledge transfer individually (which can lead to re-design of the CarePack).

It is important to note that the design of CarePacks needs to reflect the duality of routines and the unique approach by securing a balance between covering every possibility, issue and situation in a collaborative transfer of knowledge and formulating transfer routines for successful inter-organizational transfer. Additionally, the knowledge transfer profits from a better incorporation of transfer results into the periodic organizational performance reviews.

What we addressed:

We offered a creation of "How-to-Design a CarePack" to help the Coach in new designs. Furthermore, we improved the presentation of the patterns. As a communication instrument, a coherent and short presentation of a CarePack additionally stresses the value of such pattern as a portable accommodation instrument for new design paradigms discovered by collaborating teams. Especially for the collaboration within the outsourcing relationship set-ups, an ability to find a common ground for collaborating team members by proposing a one or maximum two page document, instead of a sixty page book, would be of a great value for all involved.

We also believe that the organization needs to address a structured approach to the competency mapping and competency development of the employees. This could enhance the role of the CarePacks in supporting the development of the employee; on the other hand, the competency map could be used for choosing appropriate CarePack for skills of particular knowledge receivers and identify candidates with the ability to disseminate the knowledge across the organization. 


\section{Conclusion and practical implications}

Initial efforts to support the knowledge transfer in our field project strove to determine the specific demand of the involved organizations and to design a set of routine processes for the knowledge transfer activities on the level of the knowledge transfer episode. The efforts focused on collecting proven designs for transferring knowledge such as "after action reviews" (Dixon, 2000), "peer assist" (Collison, 2004) etc. These proven designs were incorporated in the initial CarePacks. The collection of the CarePacks proved to be useful, but of course on their own they are not sufficient to fully implement the knowledge transfer. In contrast to workshops and meetings (i.e., the traditional application areas of collaboration engineering (de Vreede, 2005)), the organizational "surroundings" frequently interfered with the collaborative processes in the form of interruptions, change in priorities, lack of guidance, de-motivated employees, hidden agendas and other obstacles. In traditional workshops and meetings, moderators have the possibility to control the meeting process, motivate and focus the participants and keep interruptions away from them. Yet it is not feasible to have a moderator present each time the knowledge transfer activities were to take place. The knowledge transfer is by nature far too much embedded in work activities and having a moderator there is too expensive and sometimes may prove counter productive. Thus the task of the moderator had to be taken over by the organizational surrounding and the participants. Collaboration engineering had to design a network of actors to fulfill this task and to enable them to do the work.

Care-Packs are addressing the need for sustainable, routine-based repeatable collaboration instruments which can support the knowledge transfer during IT outsourcing relationships between organizations. Furthermore, Care-Packs are designed not only to empower teams involved in the knowledge transfer process without showing disrespect for the complexity of the transfer, but also to support individuals sharing their knowledge about the tasks, needed skills, information and know-how between the two organizations.

Based on the experience that we have gathered throughout the knowledge transfer project between the Swiss financial institution and their IT service provider, we offer important practical implications for conducting and managing transfer of knowledge during the IT outsourcing projects. Judging by the current developments in sourcing initiatives led by biggest banks (e.g., sourcing initiatives of UBS Bank) and insourcing tendencies seen in North America (Fowler, 2006), we believe that preparing organizations to successfully conduct transfer of knowledge can only gain importance in the future. We believe that there is a strong need for developing and deploying CarePacks - like instruments for the knowledge transfer in IT outsourcing - since they not only offer sustainable processes for handling the transfer, but also because the knowledge transfer routines allow the employees to develop skills important for knowledge sharing and further shape the culture of the organization. CarePacks follow the structure of patterns and can profit from qualities associated with them. Another important quality of the CarePacks is that the usage of patterns fosters development of new 
and better (for both the transferring and receiving teams) methods of the knowledge transfer in an outsourcing relationship. Finally,, such patterns provide a platform for community-based altering or creating new patterns which are of great value for communities of sourcing organizations to work on. Such platforms, in turn, enable exchange of the knowledge transfer patterns to help service receivers in balancing the knowledge with service providers during the course of IT outsourcing relationships.

If designed and implemented properly, Care-Packs could be put into practice by numerous companies as well non-profit organizations struggling to manage one of the most significant long-term risks inherent in each outsourcing relationship: erosion of knowledge and skills. It oftentimes proves to be very costly in cases where an organization needs to redefine or terminate an existing outsourcing relationship due to changes in the chosen strategy or external ramifications.

Acknowledgements: We would like to thank the project sponsor Gerhard Schwabe for valuable ideas and comments and support during the project and project member Mr. Ben Voigt for data collection and analysis, sharing his insights on the knowledge transfer process as well as providing feedback to this paper.

We thank the reviewers of this paper for their insights and feedback contributing to making this work a better tool for transferring our insights.

\section{References}

Alexander, C. (1979) The Timeless Way of Building, Oxford University Press, New York.

Alexander, C., Ishikawa, S., Silverstein, M., Jacobson, M., Fiksdahl-King, I., Angel, S., (1977) A Pattern Language: Towns, Buildings, Construction, Oxford University Press, New York.

Becker, J., Delfmann, P., (Ed.) (2004) Referenzmodellierung - Grundlagen, Techniken und domänenbezogene Anwendung, Springer.

Bloch, M., Jans, Ch., (2005) Reducing risk in offshoring projects, The McKinsey Quarterly, No.3

Braun, C., Hafner, M., Wortmann, F., (2004) Methodenkonstruktion als wissenschaftlicher Erkenntnisansatz, University of St. Gallen, St. Gallen.

Briggs, R. O., Vreede G.J., De, Nunamaker J.F, Jr., (2003) Collaboration Engineering With ThinkLets To Pursue Sustained Success With Group Support Systems, Journal of Management Information Systems, 19 (4), pp.

Bugajska, M. (2007) Piloting Knowledge Transfer in IT/IS Outsourcing Relationship. Towards Sustainable Knowledge Transfer Process. Learnings From Swiss Financial Institution., ACIS, Keystone.

Bugajska, M., Schwabe, G., Voigt, B., (2006) DEAN: Demand Analysis Method for Knowledge Transfer in IT Outsourcing Relationships, Proceedings of Third International Conference on Knowledge Management, London.

Carmel, E., Tija, P., (2005) Offshoring Information Technology, Cambridge University Press, Cambridge.

Cohen, L., Young, A., (Gartner, Inc.) (2006) Multisourcing. Moving Beyond Outsourcing to Achieve Growth and Agility, Harvard Business School Press, Boston, Massachusetts.

Collison, C., Parcell, G. (2004) Learning to Fly: Practical Lessons from one of the World's Leading Knowledge Companies, Capstone, Oxford. 
Darr, E. D., Kurtzberg, T. R., (2000) An investigation of partner similarity dimensions on knowledge transfer, Organizational Behavior and Human Decision Processes, 82 (1), pp. 2844.

Davenport, T., H., (2005) Thinking for a Living. How to Get Better Performance and Results from Knowledge Workers, Harvard Business School Press, Boston, Massachusetts.

Davenport, T., Prusak, L., (2000) Working Knowledge. How Organizations Manage what they know, Harvard Business School Press, Boston, Massachusetts.

de Vreede, G.-J., Briggs, R.O. (2005) Collaboration Engineering: Designing Repeatable Processes for High-Value Collaborative Tasks, HICSS, International Conference on System Sciences, Vol. 03, Hawaii.

de Vreede, G.-J., Kolfschoten, G.L.,Briggs, R.O. (2006) ThinkLets: a collaboration engineering pattern language, International Journal of Computer Applications in Technology, 25 (2/3), pp. $140-154$.

Dixon, N. (2000) Common Knowledge. How Companies Thrive by Sharing what They Know., Harvard Business School Press, Boston, Massachusetts.

English, W., Baker, W., H., Jr. (2006) Winning the Knowledge Transfer Race, McGraw-Hill, New York.

Fowler, B. (2006) Is Insourcing the New Outsourcing? CIO.

Gamma, E., Helm, R., Johnson, R., Vlissides, J., (1995) Design Patterns, Elements of Reusable Object-Oriented Software, Addison-Wesley.

Gottschalk, P. (2007) Knowledge Management Systems: Value Shop Creation, IGI Publishing.

Gregor, S. (2006) The Nature of Theory in Information Systems, MIS Quarterly, 30 (3), pp. 611656.

Heinzl, A. K., W.; Hack, J., (2001) Erkenntnisziele der Wirtschaftsinformatik in den nächsten drei und zehn Jahren, Wirtschaftsinformatik, 43 (3), pp. S. 223-233.

Heym, M. (1993) Methoden-Engineering: Spezifikation und Integration von Entwicklungsmethoden fuer Informationssysteme, Rosch-Buch, Hallstadt.

Hinds, P., Pfeffer, J., (2003) Why Organizations Don't "Know What They Know": Cognitive and Motivational Factors Affecting the Transfer of Expertise., In Sharing Expertise. Beyond Knowledge Management(Ed, Ackerman, M., S., Pipek, V., Wulf, V.) MIT Press, Boston.

Hutzschenreuter, T. (2004) Evaluator for knowledge transfer, Otto Beisheim Graduate School of Management.

Jensen, M. M., W., (1976) Theory of the firm: Managerial behavior, agency costs and ownership structure, Journal of Financial Economics, (3), pp. 305-360.

Kim, L., Nelson, R.R., (2000) Technology, Learning and Innovation: Experiences of Newly Industrializing Economies., Cambridge University Press, Cambridge, UK.

Ko, D., Kirsch, L., J., King, W.,R. (2005) Antecedents of Knowledge Transfer from Consultants to Clients in Enterprise System Implementations, MIS Quarterly, 29 (1), pp. 59-85.

Lukosch, S., Schümmer, T. (2006) Groupware development support with technology patterns, International Journal of Human-Computer Studies, 64 (7), pp. 599-610.

Mulder, U., Whiteley, A., (2007) Emerging and capturing tacit knowledge: a methodology for a bounded environment, Journal of Knowledge Management, 11 (1), pp. 68 - 83.

Nonaka, I., Takeuchi, H., (1995) The Knowledge-Creating Company. How Companies Create the Dynamics of Innovation, Oxford University Press, New York, Oxford.

Owen, C. (1997) Design Research: Building the Knowledge Base, Journal of the Japanese Society for the Science of Design, 5 (2), pp. 36-45.

Pavitt, K. (2002) Innovating routines in the business firm: what corporate tasks should they be accomplishing? Industrial and Corporate Changes, 11 117-123.

Rulke, D. L., Zaheer, S, Anderson, M., (2000) Sources of managers' knowledge of organizational capabilities, Organizational Behavior and Human Decision Processes, 82 (1), pp. 134-149.

Szulanski, G. (1999) The process of knowledge transfer: A diachronic analysis of stickiness, Organizational Behavior and Human Decision Processes, 82 (1), pp. 9-27. 
Thatchenkery, T. (2005) Appreciative Sharing of Knowledge: Leveraging Knowledge Management for Strategic Change, Taos Institute Publications, Chagrin Falls, Ohio.

Von Krogh, G., (1998) Der Wissenstransfer in Unternehmen: Phasen des Wissenstransfers und wichtige Einflussfaktoren, Die Unternehmung, 52 (5), pp. 235-253.

Voigt, B., Novak, J., Schwabe, G., (2007) How to manage knowledge transfer in IT outsourcing relationships - towards a reference model, AMCIS, Keystone, Colorado.

Willcocks, L., P., Lacity, M., C., (2006) Managing knowledge in outsourcing: Cases in financial services., In Global Sourcing of Businesses and IT Services(Ed, Willcocks, L., P., Hindle, J., Feeny, D.,Lacity, M., C.) Palgrave Macmillan, New York.

WKWI (1994) Profile of Information Systems Research, Wirtschaftsinformatik, 36 (1), pp. S. 8081. 